\title{
Proyecto de baile flamenco: desarrollo motriz y emocional en educación infantil Flamenco dance project: motor and emotional development in early childhood education
} Rosario Padial-Ruz, Delia Ibáñez-Granados, Marina Fernández Hervás, José Luis Ubago-Jiménez Universidad de Granada (España)

\begin{abstract}
Resumen. El presente proyecto educativo, que tiene como eje central el baile flamenco, muestra una experiencia didáctica con alumnado de educación infantil, con el objetivo de utilizar el baile como recurso didáctico para el desarrollo motriz y emocional. A lo largo del proyecto se mantuvo una metodología adecuada a la edad, al contexto y al objetivo marcado desde el inicio del mismo. Se utilizaron recursos materiales tanto reciclados como los propios del aula y otros relacionados directamente con el flamenco. Los resultados muestran la posibilidad de trabajar el baile flamenco como recurso didáctico en el aula de educación infantil de manera lúdica. Por lo que se concluye que utilizar este recurso desde edades tempranas, proporciona una mejora integral del alumnado.
\end{abstract}

Palabras clave. Baile flamenco, educación infantil, desarrollo motor, emoción, educación integral.

Abstract. The present educational project, which has flamenco dance as its central axis, shows a didactic experience with pupils of early childhood education, with the aim to use dance as a didactic resource for motor and emotional development. Throughout the project, a methodology tailored to age, context, and to the objectives set at the beginning was maintained. Either recycled resources, material from the classroom, and other appliances strictly related to flamenco were employed. The results show that flamenco dance can be used effectively as a didactic ludic tool in early childhood education. Therefore, using this resource from early ages contributes to students' integral development.

Keyword. Flamenco dancing, early childhood education, motor development, emotion, integral education.

\section{Introducción}

En el marco de la ley queda explicitada la importancia de que el Sistema Educativo fomente el desarrollo integral del alumnado, para lo que es necesario satisfacer todos los ámbitos, físico, motriz, emocional, social y cognitivo (Gil-Espinosa, Romance \& Nielsen, 2018).

Asímismo, la relevancia del juego y del movimiento en el desarrollo infantil, queda reflejada en la necesidad de actividad física en estas edades, para una buena salud integral de los niños, siendo las recomendaciones de un mínimo de 60 minutos diarios de actividad física moderada o vigorosa (OMS, 2010) y en el horario escolar, de al menos 180 minutos de actividad física semanal,según la UNESCO (2015).Así, se convierten en una parte fundamental para el desarrollo integral del alumnado. Al no estar recogida con un horario concreto, dado el carácter global de la etapa, es conveniente diseñar metodologías activas, basadas en el juego y el movimiento,que cubran estos mínimos y prevengan de enfermedadesrelacionadas con el sedentarismo,como la obesidad (GilEspinosa, et al., 2018).

Los beneficios de la utilización de metodologías activas en edades tempranas, utilizando el juego y el movimiento como eje transversal para la enseñanza integral de los diferentes contenidos recogidos en los currículos, son numerosos y han sido comprobados recientemente en diferentes estudios,en los que se han obtenido mejoras de la condición física, habilidades motoras, promoción del aprendizaje, educación integral, prevención del sedentarismo y la salud psicosocial(disfrute, motivación, creatividad, autonomía, autoconcepto, relación con los demás y desarrollo de emociones positivas en las actividades y juegos de tipo cooperativo), motivación e interés hacia los contenidos de trabajo de la actividad, entre otras (Duran \& Costes, 2018 ;Gil-Espinosa, et al., 2018; Moreno-Murcia, Huéscar, Polo, López, Carbonell \& Meseguer, 2016;Padial\& Sáenz-López, 2014;Ríos, Navarro, Arufe \&Pérez, 2018; Teixeira, Abelairas-Gomez, Arufe-Giráldez, Pazos \&BarcalaFurelos,2015; Zamorano, Gil-Madrona, Prieto-Ayuso \& Zamorano, 2018).

Sin embargo, en la práctica, seguimos observando quela educación motriz no es tratada con la importancia que requierela etapa infantil (Gil-Espinosa, et al.,2018; Viciana, Cano, Chacón, Padial\& Martínez, 2017), dado que la mayor parte del tiempo es dedicado a las materias académicas que recoge el currículum clásico (matemáticas, inglés, len-

Fecha recepción: 12-02-18. Fecha de aceptación: 27-09-18 Rosario Padial Ruz rpadial@ugr.es gua...), quedando en muchas ocasiones al margen de la formación del alumnadolas áreas transversales con un tiempo más limitado. Pese a que diferentes estudios realizados sobre las perspectivas del alumnado y profesorado del Grado de Educación infantil, en cuanto a la utilización de la motricidad en el aula, reflejan un gran interés y valoración de su importancia para el desarrollo integral de los niños,datos actuales denotan una baja preparación del profesorado y una posterior escasa aplicación de la misma en el aula de infantil (Solís, Prieto, Nistal \& Vázquez, 2017; Valdemoros-San Emeterio, Sanz-Arazuri, Ponce-de-León-Elizondo \& Alonso-Ruiz, 2018). Esto pone de manifiesto la necesidad de fomentar en los centros educativos el trabajo de la motricidad, la expresión y comunicación en el alumnado y de una mayor formación en motricidad del profesorado de la etapa, considerando incluso la incorporación de especialistas en la materia:

La importancia del juego y la actividad física en el desarrollo infantil sugiere la necesidad de incorporar profesorado especialista en Educación Física en dicha etapa con la intencionalidad de mejorar el desarrollo integral del alumnado y garantizar la utilización del tiempo necesario en horario escolar y curricular (Gil-Espinosa, et al., 2018, p.256).

Una de las manifestaciones básicas de la motricidad es la danza, que se caracteriza por el uso de metodologías innovadoras y no directivas, que desarrollan la creatividad, la imaginación y la capacidad de deshinibición del alumnado (Rial \&Villanuena, 2013).

Los beneficios que puede aportar la danza en general son muchos, pero entre todos hemos de destacar la oportunidadde satisfacer las necesidades de expresión y creación, a través de una cadena de movimientos creados con su propio cuerpo (Cañal \& Cañal,2001; Rial \& Villanueva, 2016).

Si la danza se integra en el ámbito educativo, tal y como reflejanVicente, Ureña, Gómez\&Carrillo (2010), el alumnado desarrollará habilidadesde coordinación, perceptivo-motoras, control y conocimiento corporal, lo que le permitirá expresarse con un nuevo lenguaje de manera personal. Se acercará al mundo de la danza, así como, favorecerá su socialización e interrelación con sus iguales.Serárelevante en el desarrollo intelectual, afectivo y emocional del alumnado (de Rueda \& López, 2013;Fuentes, 2006; Rizo, 1996), mejorará su autoestima, obteniendo beneficios en relación a la felicidad-satisfacción (Abilleira, FernándezVillarino, Ramallo\& Prieto, 2017) y mejorará su conocimiento y diversidad cultural (Liu, 2017), en definitiva y desde el campo de la motricidad y de la salud física, mental y emocional, es una forma de estrategia para su mejora y promoción (López, Carlson, Cain, Bonilla, Chuang, Elder \&Sallis, 2015).

Dentro del ámbito educativo, es un medio innovador, propicia un 
ambiente diferente de aprendizaje, permite exteriorizar sensaciones, emociones y sentimientos a través del cuerpo, es una posibilidad de aprendizaje, expresión y comunicación con sus iguales y facilita el desarrollo de los diferentes contenidos: conceptuales, procedimentales, y actitudinales (Renobell, 2009), mejora los niveles de aprendizaje del alumnado y la calidad de la enseñanza (Liu, 2017).

En la escuela se puede iniciar al alumnado en distintos estilos de danza, considerando que el principal medio para trabajarlo es a través del juego, intentando que éste se divierta, se interese y se motive aprendiendo, dándole así otra alternativa para sunecesidad de movimiento, de expresión y de poder mostrar sus emociones (de Rueda \& López, 2013; Esteve \&López,2014; Rial \& Villanueva, 2016).

Dada la existente relación entre la danza y la educación integral en educación infantil,se plantea un nuevo enfoque para el trabajo de la motricidad, donde el baile flamenco se utilice como recurso didáctico, acercando al alumnado al patrimonio cultural más cercano de una forma lúdica y adecuada a un óptimo desarrollo de su creatividad.

El flamenco es una seña de identidad de nuestra cultura, a nivel nacional y concretamente en la comunidad deAndalucía, donde el Gobierno establece medidas legales para su inclusiónen el sistema educativo, recogidasen la Ley de Educación deAndalucía, artículo 40. El currículo deberá contemplar la presencia de contenidos y de actividades relacionadas con el medio natural, la historia, la cultura y otros hechos diferenciadores de Andalucía, como el flamenco, para que sean conocidos, valorados y respetados como patrimonio propio y en el marco de la cultura española y universal (Pardo \& Pacheco-Álvarez, 2014).

El aprendizaje del arte y la cultura en los centros educativos constituye una de las estrategias más poderosas para la construcción de una ciudadanía intercultural. Además, la presencia del Flamencoen el sistema educativo a través de las diferentes áreas de conocimiento, contribuye al desarrollo integral y pleno del alumnado, además de favorecer la valoración, apreciación y respeto por este arte...para una parte de la ciudadanía para la que era desconocido. La valoración social del Flamenco y el rango que éste ha alcanzado como manifestación cultural deben tener su reflejo en el sistema educativo andaluz, por lo que parece conveniente establecer medidas para la inclusión del Flamenco en el sistema educativo andaluz (BOJA, 2014, p. 17).

Entre las medidas que se proponen se encuentra la inclusión del flamenco en la planificación de actividades extraescolares y complementarias del centro, Sin embargo, es rara su utilización en el aula:

Es un arte declarado en el 2010 Patrimonio Inmaterial de la Humanidad por la Unesco. De esta forma, la comunidad internacional reconoce cómo los sentimientos más profundos de alegría, tristeza, angustia, soledad, dolor, amor, desamor y pasión son conducidos a través de su música mediante cada uno de los «palos flamencos». Parece contradictorio entonces que su inclusión en nuestras aulas aún sea una asignatura pendiente (de las Heras \& Calderón-Garrido, 2018, p.54).

El flamenco es un tipo de baile que genera un entorno lúdico de aprendizaje, estimulando la espontaneidad y mejora del desarrollo y bienestar del alumnado (Jefferson-Buchanan, 2012), también se utiliza en las aulas intentando favorecer el bienestar y el rendimiento del alumnado en las mismas. Si se ofrecen experiencias positivas y de afecto mientras los niños experimentan a través de los movimientos expresivos, desarrollarán la conciencia somática y social, además de las habilidades de reconocer, expresar y regular sus propias emociones (Thom, 2010).

La práctica de este baile posee una gran importancia en el desarrollo integral delalumnado en edades tempranas. Cuando se hace una práctica continuada de esta disciplina,el alumnado desarrolla de forma óptima capacidadesfísicas, mentales y emocionales.Por otro lado, diversas experiencias reflejan los beneficios de este recurso desde el punto de vista de la integración e inclusión de alumnado de étnia gitana (García \& Lizaso, 2012) y de alumnado con necesidades educativas especiales (Pardo \& Pacheco-Álvarez, 2015). Es por tanto,que la danza, siendo un elemento educativo esencial para el desarrollo global del alumnado, sea valorada y conocida como un recurso con un gran potencial educativo.

\section{Planteamiento didáctico-experiencia}

El presente proyecto educativo se desarrolla en un centro de educación infantilde la provincia de Granada (España), con alumnado de segundo ciclo de educación infantil, con edades comprendidas entre los tres y cinco años.

Teniendo en cuenta la importancia y necesidad de mejorar el tiempo de práctica motriz para prevenir y mejorar desde el aula el sedentarismo y la obesidad infantil, aportamos un recurso para la educación integral del alumnado de la etapa, basado en la motricidad como eje transversal. Se pretende, utilizando recursos basados en el cuerpo y el movimiento, que el alumnado adquiera aprendizajes en las tres dimensiones: conocimiento de sí mismo y autonomía personal, en el conocimiento del entorno y en el lenguaje, en su relación con la comunicación y representación.

La utilización en concreto del baile flamenco como recurso, surge a raíz de observar una gran carencia en su utilización en el aula como recurso educativo y por los beneficios que aporta en los diferentes ámbitos de desarrollo del alumnado.

Partiendo de la Orden del 5 de Agosto de 2008, por la que se desarrolla el Currículo correspondiente a la educación infantil en Andalucía, el flamenco se ve reflejado en varios de sus apartados, así en sus objetivos generales se establece que el docente debe ser facilitador, y dar a conocery participar en las manifestaciones culturales y artísticas de Andalucía y la pluralidad cultural, fomentando el respeto e interés hacia las diferentes culturas y la propia.

La presencia del flamenco se refleja en las diferentes áreasdel currículo:

- Conocimiento de sí mismo y autonomía personal. En esta área de conocimiento se crea la identidad del alumnado y se genera su autonomía personal mediante la interacción con el entorno social, físico y cultural, de manera que los centros fomentan el contacto y el encuentro con personas adultas y niños de diversos orígenes culturales, diferentes religiones, etnias, costumbres, hábitos... haciendo de la diversidad un campo privilegiado de la experiencia educativa. En este caso, trabajamos el flamenco, principalmente a través del baile.

- Conocimiento del entorno.El conocimiento del entorno supone ofrecer al alumnado vivir situaciones afectivas y sociales en distintos contextos, acercándolos a producciones culturales propias y disfrutando de ellas con experiencias nuevas relacionadas con ese medio físico, natural y social. El alumnado consigue este conocimiento mediante la manipulación, observación, indagación, exploración, comprobación, modificación, verbalización y representación. Captando informaciones y construyendo su propia realidad.En este proyecto se vincula al alumnado al entorno cultural participando activamente en situaciones y manifestaciones culturales de nuestro medio, acercándolos a los estilos de vida, a las fiestas y celebraciones y a las producciones culturales y folklore andaluz, percibiendo así la pluralidad cultural presente actualmente en la sociedad. Por ejemplo, podemos conocer el día internacional del flamenco, así como las fiestas tradicionales de Granada, día de la Cruz y el Corpus Christi.

-Lenguajes: comunicación y representación.Se trabajará el reconocimiento de lenguajes y expresiones de las diversas realidades multiculturales en las que se encuentra el alumnado. La educación sensorial y artística, son lenguajes de enriquecimiento cultural mediante los cuales, el alumnado conocerá las tradiciones culturales propias y ajenas. La música es el medio principal que usaremos para dar lugar a la comunicación y representación de las distintas necesidades o emociones que el alumnado desee expresar. Una herramienta fundamental en ésta área, es su propio cuerpo, utilizando la danza flamenca como medio de comunicación, representación y expresión.

\section{Objetivos y contenidos del proyecto}

En función de lo anteriormente mencionado, los objetivos de este proyecto fueron: 
- Conocer y practicar diferentes bailes flamencos, utilizándolo como un recurso para el desarrollo expresivo, motriz, afectivo-social y de salud física y mental.

- Identificar los «palos» más característicos del flamenco.

- Conocer y manipular diferentes elementos que están directamente relacionados con la danza flamenca como instrumentos musicales, objetos del baile flamenco, etc.

- Mejorar las habilidades personales, sociales, emocionales y motrices a través de las actividades motrices planteadas.

Los contenidos trabajados fueron:los bailes flamencos y sus diferentes palos; personajes famosos del baile flamenco; accesorios que usan los bailaores y bailaoras en sus actuaciones; cultura del flamenco; exteriorización de emociones a través del baile flamenco; motricidad y expresión a través del baile flamenco e interpretación de bailes flamencos.

\section{Metodología}

Para la experiencia se utilizó una metodología activa e integradora, primando el cuerpo y el movimiento como ejes para el aprendizaje global del resto de contenidos que se presentan en la propuesta.Para ello se han utilizados recursos didácticos como el juego y la expresión corporal, el aprendizaje basado en proyectos, talleres, juegos (creativos, libres y de tipo cooperativo) y la danza, debido a su versatilidad para desarrollar distintas capacidades (Mérida-Serrano, Olivares-García \& González-Alfaya, 2018) y cuyo producto final fue la celebración del día internacional del flamenco el 16 de febrero.

La metodología basada en proyectos posibilita trabajar desde el principio de globalización de los contenidos y reúne rasgos comunes al enfoque de la motricidad en la etapa, ya que permite un aprendizaje activo, motivador, cooperativo y basado en el niño. Por otro lado, la utilización de los talleres como recurso metodológico se centra en la posibilidad que ofrecen para desarrollar actividades manipulativas, como la construcción del vestuario de flamenco, que posteriormente utilizaremos para favorecer el desarrollo motriz y expresivo del alumnado (Mérida-Serrano, et al., 2018).

Las orientaciones metodológicas que guiaron el proyecto, se basaron en laorden del 5 de Agosto de 2008, teniendo en cuenta el principio de globalización, así el alumnado del segundo ciclo de infantil abordó experiencias de aprendizaje poniendo en juego mecanismos afectivos, intelectuales y expresivos.

Las diferentes actividades y talleres se llevaron a cabo durante los meses de septiembre, octubre y noviembre, como se indica a continuación, atendiendo a los principios metodológicos de atención a la diversidad, globalización de contenidos, aprendizaje significativo, socialización, creación de ambientes, organización del espacio y el tiempo, utilización del juego como instrumento de motivación y aprendizaje, observación y experimentación.

En relación a los recursos que hemos necesitado a lo largo de todo el proyecto podemos destacar:

- Recursos materiales. Para la realización de la mayor parte de los talleres, se utilizaron materiales tanto reciclados (cartones, botellas, diferentes tipos de papel,envases...) como los propios del aula (pegamentos, tijeras, ceras, rotuladores, pintura de dedos...). También se necesitaron otros tipos de materiales relacionados directamente con el flamenco como: cds de música, vestuario de baile flamenco, macetas al estilo andaluz, telas de lunares, abanicos, flores...Además de juegos de mesa creados por el docente y flash card (de palabras y de fotografías).

- Recursos físicos o espaciales. Tales como el aula, el gimnasio y el patio.

- Recursos tecnológicos, como reproductores de música y de video. Necesarios para la escucha de los diferentes palos flamencos y la visualización de actuaciones flamencas interpretadas por artistas.

- Recursos humanos.Se contó con las familias, que ayudaron en todo momento a la consecución de los objetivos marcados por el proyecto, y el apoyo de profesionales en el ámbito de la danza que poseían experiencia docente con niños de edad infantil.
- Recursos económicos. Se centraron en las posibilidades de la mayor parte de las familias. Cada uno aportó materiales dentro de sus posibilidades, y se priorizó la utilización de materiales reciclados para abaratar el proyecto.

\section{Temporalización}

El desarrollo del proyecto se realizó durante los meses de septiembre, octubre y noviembre de 2017. Su organización se estructuró en tres días a la semana (lunes, miércoles y viernes), variando la duración y forma de las actividades.Previamente se desarrolló un cronograma donde se planificó las diferentesintervenciones que se llevarían a cabo, designado una letra en orden alfabético a cada sesión, como se muestra a continuación:

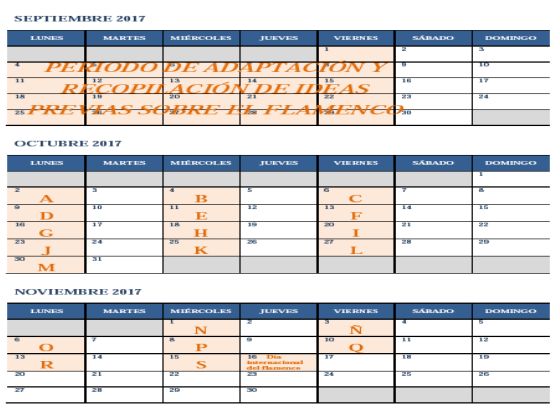

Figura 1.

Cronogramade la organización y distribución de sesiones en orden alfabético, durante el desarrollo del proyecto. Elaboración propia.

En el mes de septiembre:Se inició una fase previa de conocimiento e información a padres y alumnado sobre el proyecto y la utilización del flamenco como un recurso didáctico dentro del aula. Por otro lado se realizó el diseño de las diferentes actividades que fueronutilizadas durante el desarrollo del mismo.

En el mes de octubre: Se iniciaron las diferentes actividades con el alumnado, que continuaron durante el mes de noviembre hasta el día internacional del flamenco. Estas fueron estructuradas y desarrolladas de forma paralela, diferenciadas en tres grupos:

Grupo 1.Talleresmotrices y plásticos, con un total de siete sesiones (E, K, M, N, O, P y R). Grupo 2.Actividades complementarias. En las que se trabajaron los valores, las emociones, los aspectos culturales....con un total de siete sesiones (A, B, D, G, H, J y S) y Grupo 3. Taller de elaboración del baile flamenco. Aprendizaje y ensayo de la coreografía con una duración de siete sesiones (C, F, I, L, Ñ, Q, y día internacional del flamenco16 de noviembre).

\section{Desarrollo de los talleres y actividades realizadas}

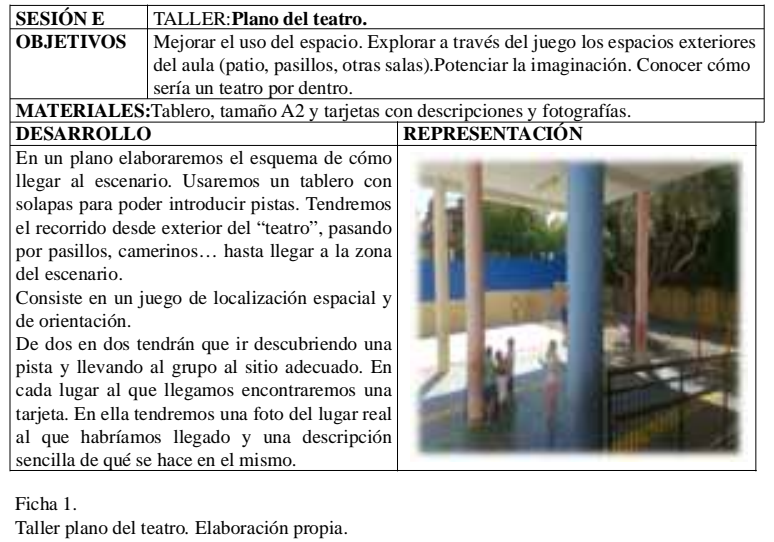




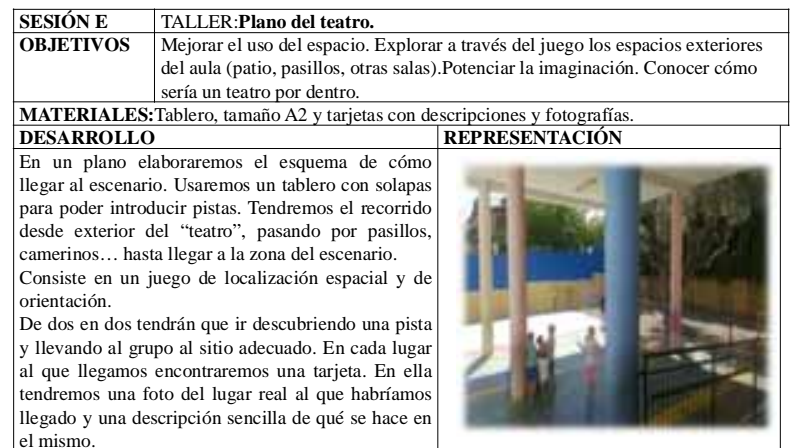

llegado y una descripción sencilla de qué se hace e

el mismo.

Taller plano del teatro. Elaboración propia.

\begin{tabular}{|l|l|}
\hline SESIÓN K & TALLER: Emocionario flamenco. \\
\hline
\end{tabular}

\begin{tabular}{|l|l|}
\hline OBJETIVOS & Reconocery expresar emociones. Imitar movimientos. Desarrollar la \\
\hline
\end{tabular}

MATERIALES:Equipo de reproducción y visualización, ordenador, altavoces y proyector.

\begin{tabular}{|l|}
\hline DESARROLLO \\
\hline Haremos una asamblea donde visualizaremos
\end{tabular}

Haremos una asamblea donde visualizaremos
en el proyector vídeos de artistas flamencos

en el proyector vídeos de artistas flamencos
bailando diferentes palos. Los videos serán

bailando diferentes palos. Los videos serán
cortos e intentaremos que sobresalga la

emoción que queremos trabajar. Cuando haya

terminado el primer video preguntaremos a

los niños qué emoción expresaba el bailaor o

la bailaora. El niño que conteste de forma correcta le pediremos que imite a ese artista. Por ejemplo, visualizamos unas alegrías bailadas por Manuel Liñann, quien acierte la emoción intentará imitar los movimientos que
le han resultado alegres o que le han gustado. le han resultado alegres o que le han gustado. Ficha 2. REPRESENTACIÓN

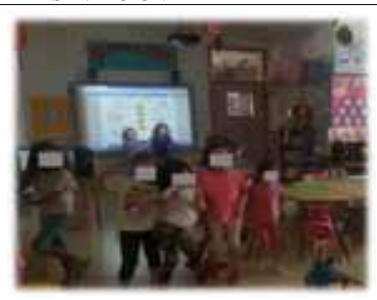

Taller emocionario flamenco. Elaboración propia.

\begin{tabular}{|l|l|}
\hline SESIÓN M & TALLER: Disfrutamos en familia. \\
\hline
\end{tabular}

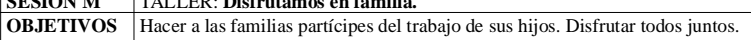
te elementos usados para el baile flamenco Colaborar en la elaboración.
con materiales reciclados.

MATERIALES:Bolsas de basura, cartones, botellas, papeles de todo tipo, colores, pinturas, tapones... Todos los materiales reciclados que las familias deseen emplear en sus talleres.

\begin{tabular}{l|l} 
tapones...Todos los materiales reciclados que las fa \\
\hline DESARROLLO \\
\hline Las familias que quieran participar tendrán que \\
\hline
\end{tabular} Las tamilias que quieran patticipar, tendrán que elegir un elemento usado en el baile flamenco, para

feria flamenca, durante melia maña ca

feria flamenca, durante media mañana. Cada familia tendrá asignada tna mesa (taller). E talleres. Conoćn jos elementos uso baile flane baile flancenco donde se relizá el baile. El gúa ma los don pasos y los ninos en forna de espejo tendrán que bailar tal y cono e guía lo ma. Dejando de la canción para baile libre o improvisación.

Ficha 3.

Taller disfrutamos con la familia. Elaboración propia.

\begin{tabular}{|l|l|l|}
\hline SESIÓN N & TALLER:Caracterización I. \\
\hline
\end{tabular}

\begin{tabular}{|l|l|}
\hline OBSETIVOS & TALLER:Caracterización I. \\
\hline Aprender a usar materiales reciclados. Conocer los patrones de algunos trajes
\end{tabular} flamencos. Desarrollar la imaginación. Elaborar el elemento de la vestiment flamencos. Desarrollar la
flamenca que más le guste.

flamenca que más le guste.
MATERIALES:Todos los materiales reciclados que podamos utilizar. Bolsas, cartones, MATERIALES:Todos los materiales reciclados que pod
cartulinas, plásticos, pinturas, colores, tijeras, pegamento y cola.

cartulinas, plásticos, pinturas, colores, tijeras \begin{tabular}{|l|}
\hline DESARROLLO \\
\hline De forma individual cada alumno elaborar
\end{tabular} De forma individual cada alumno elabora
una prenda flamenca, serán libres de elegir $\begin{array}{llll}\text { entre chalecos, faldas, } & \text { camisas, } \\ \text { pantalones... y elaborarán su } & \text { traje al }\end{array} \mid$ $\begin{aligned} & \text { pantalones... } \\ & \text { completo. }\end{aligned}$ y elaborarán su traje al
a completo.

Nota: el guía tendrá patrones adecuados de forma general al alumnado para que ellos mismos sigan el patrón y puedan trabajar de orma independiente.

Ficha 4.

Taller caracterización I. Elaboración propia.

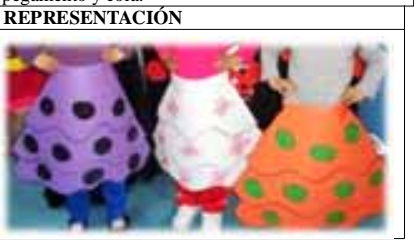

\begin{tabular}{|l|l}
\hline SESIÓN O & TALLER:Caracterización II.
\end{tabular}

OBJETIVOS Desarrollar la imaginación. Fabricar complementos de flamenco usando materiales reciclados. Mejorar el compañerismo y el cariño entre compañeros. Expresar lo que sienten sobre sus compañeros.

MATERIALES:Papeles de varios tipos (charol, pinocho, cartulina), pegamentos, materiales reciclados, cartones, tetrabrik, botellas.

DESARROLLO

Con materiales que tenemos en el colegio además de algunos materiales reciclados, elaboraremos complementos para su traje flamenco. Por ejemplo, flores, sombreros, abanicos, collares, pulseras. .

Por parejas, alumno preguntará al compañero que complemento prefiere. Cada uno confeccionará el que le gusta a su compañero. Al final de la actividad cad niño en un trocito de papel le escribirá o expresará la emoción que ha sentido al realizarle ese complemento y dos adjetivos positivos que le gusten de su compañero.

Ficha 5 .

Taller caracterización II. Elaboración propia. \begin{tabular}{|l|l|}
\hline SESIÓN P & TALLER:Caracterización III. \\
\hline OB
\end{tabular}

\begin{tabular}{l|l}
\hline OBJETIVOS & Aprender a usar materiales reciclados. Desarrollar la imaginación. Propiciar un \\
\hline
\end{tabular} ambiente de compañerismo y cariño entre amigos. Elaborar un mantón.

MATERIALES:Telas de $1 \mathrm{~m} \times 1 \mathrm{~m}$, tijeras y pistola termo fusible, pintura especial para telas, formas para estampar, lana o hilo grueso $\mathrm{y}$ toallitas.

DESARROLLO

REPRESENTACIÓN

La actividad comenzará con la visualización de dos

videos, uno del artista Manuel Liñán, bailando con bata de cola y mantón. El segundo video será también de una artista reconocida, Blanca Del Rey, bailando con mantón.Después pediremos opiniones de cual le gusta más o menos, que le transmiten... Con pintura para telas, estamparemos diferentes formas, hojas, flores, lunares... de colores en cada uno de los mantones. Por equipos de trabajo.Primero de forma individual estampando con pintura.Luego de forma cooperativa cada uno firmará el mantón de cada uno de sus compañeros.Una vez que la pintura está seca con trozos de lana, hilo grueso u otro tipo de material, le haremos los flecos $y$ los iremos pegando con la pistol termofusible.

Ficha 6 .

Taller caracterización III. Elaboración propia.

\begin{tabular}{|l|l|}
\hline SESIÓN R & TALLER:Improvisación. \\
\hline
\end{tabular}

\begin{tabular}{|l|l}
\hline OBJETIVOS & Disfrutar bailando. Desarrollar la creatividad y espontaneidad. Mejorar la unión
\end{tabular} del grupo.

MATERIALES:Reproductor de audio.

\begin{tabular}{l|l}
\hline DESARROLLO & REPRESENTACIÓN \\
\hline
\end{tabular}

\begin{tabular}{lll}
\hline La actividad consiste en bailar \\
libremente diferentes
\end{tabular}

libremente diferentes palos de

parejas, en grupo, de forma individual.

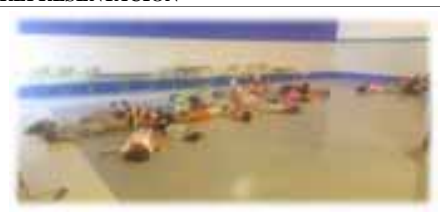

Ficha 7.

Taller improvisación. Elaboración propia.

\begin{tabular}{l|l}
\hline SESIÓN A & ACTIVIDAD COMPLEMENTARIA: ¿Qué sabemos?
\end{tabular}

OBJETIVOS Reconocer diferentes elementos que están directamente relacionados con la danza flamenca.Transmitir individualmente las ideas que tenemos sobre el flamenco en general.

MATERIALES:Flores, peinetas, pendientes, chaleco o traje de flamenco, faldas y vestidos, cenes y botas, bastón, mantón, plato de cerámica, castañuelas o instrumentos que haya en e centro, discos de cantaores, fotos de bailaores/as.

Usaremos todos aquellos materiales que tengamos a nuestro alcance.

DESARROLLO

Llevaremos al aula elementos característicos

del flamenco. Las guardaremos en una caja

"nuestro baúl flamenco". Los niños

experimentarán con todos esos elementos,

con música flamenca de fondo. Cuando

todos hayan experimentado con los

elementos flamencos explicaremos para que

sirve cada uno de ellos.

Ficha 8.

Actividad complementaria ¿Qué sabemos? Elaboración propia.

\begin{tabular}{|l|l|}
\hline SESIÓN B & ACTIVIDAD COMPLEMENTARIA:Dibuja tu vestuario. \\
\hline
\end{tabular}

OBJETIVOS Desarrollar la creatividad del alumno. Recordar los trajes flamencos que

hemos trabajado en clase. Conocer los elementos básicos del vestuario.

MATERIALES:

Se imprimirá una foto de la cara de cada niño y ellos la recortarán, posteriormente la pegaremos en vin folio y dibujarán como quieren que sea su aparezcan recordando los trajes que hemo trabajado antes.

Ficha 9 .

Actividad complementaria dibuja tu vestuario. Elaboración propia.

\begin{tabular}{|l|l|}
\hline SESIÓN D & ACTIVIDAD COMPLEMENTARIA: ¿Qué siente? \\
\hline
\end{tabular}

OBJETIVOS Relacionar las expresiones corporales con las emociones correspondientes. Conocer artistas representativos del mundo flamenco en Granada.

MATERIALES: 12 tarjetas para cada equipo: 6 fotografías de artistas flamencos, los cuales su actitud corporal y expresión facial muestran emociones diferentes; 6 tarjetas, cada una con una palabra que expresa una emoción determinada.

Un total de 48 tarjetas. Las fotografías serán diferentes en cada equipo, pero las tarjetas con los nombre de las emociones serán las mismas. Las seis emociones elegidas serán: alegría, miedo, tristeza, coraje, calma, furia.

DESARROLLO

El alumnado estará organizado en cuatro equipos de

trabajo, sentados en sus sillas. Cada equipo tiene 12

tarjetas, 6 de ellas con fotografías (que en cada equipo

pueden ser diferentes) y las 6 restantes muestran

palabras relacionadas con las emociones (en los cuatro

equipo serán las mismas, aunque las fotografías cambien).

Es un juego de hacer parejas. Una carta lleva una foto de un artista bailando, tocando la guitarra, dando palmas... y tienen que relacionarlo con otra carta que tiene el nombre de un sentimiento. La actitud corporal y la expresión facial que muestran las fotos serán determinantes para elegir que emoción están intentando transmitir.

Una vez que han unido las parejas, en asamblea analizaremos las fotos y el alumnado será protagonista de una escenificación de imitación de las imágenes.

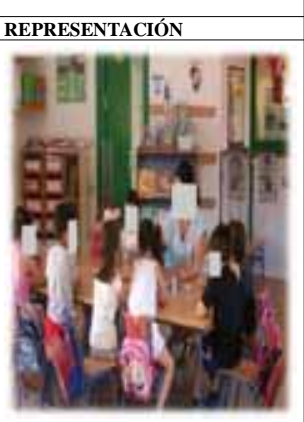

Ficha 10.

Actividad complementaria ¿Qué siente? Elaboración propia. 


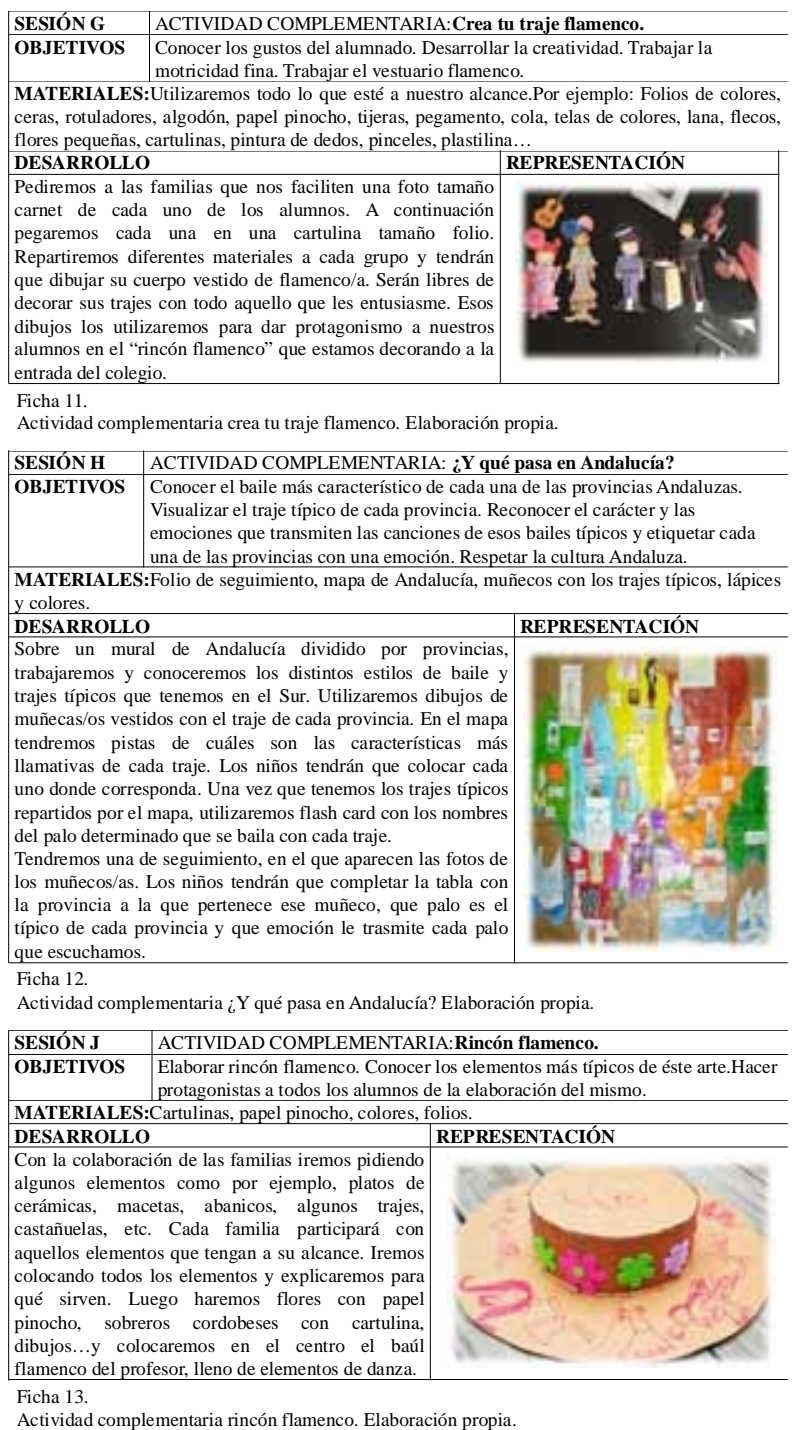

Actividad complementaria rincón flamenco. Elaboración propia.

\section{Resultados más relevantes}

La valoración que hacemos sobre los objetivos que se han conseguido en el proyecto, realizada a través de la observación sistemática, revisión de las tareas y el diario de la profesora encargada del mismo, es bastante positiva.

A través de las diferentes actividades y talleres, han adquirido conocimientos relacionados con el baile flamenco y la cultura que lo envuelve.

Con la interpretación del baile no solo contribuyó al trabajo de contenidos psicomotrices de la etapa, sino que sirvió como motivación, creando un ambiente afectivo y de integración donde todos participaron. Aunque al inicio, a los chicos les costaba implicarse en las actividades de baile, el utilizar otros recursos como el juego, hicieron que al final del proyecto estas también les resultaran atractivas.

Las actividades donde mayor interés hemos observado, han sido las relacionadas con el trabajo emocional, concretamente las actividades «qué siente» y el «emocionario flamenco» y por otro lado, aquellas en las que involucraba a la familia.

La participación de la familia fue alta (80\%) en la colaboración para aportar los materiales del «rincón flamenco», sin embargo fue mucho más escasa en la actividad»disfrutar en familia», previsiblemente por coincidir con el horario laboral de los padres.

Por último, y en cuanto a uno de los objetivos principales del proyecto, el diseño de propuesta permitió un aumentodel tiempo de práctica motriz y expresiva durante la aplicación del mismo.

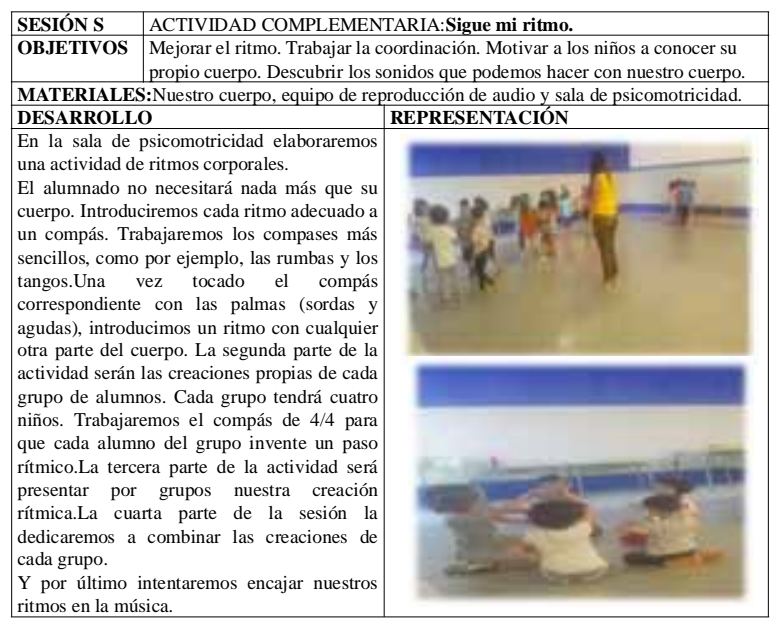

Y por último intentaremos encajar nuestros

Ficha 14.

Actividad complementaria sigue mi ritmo. Elaboración propia.

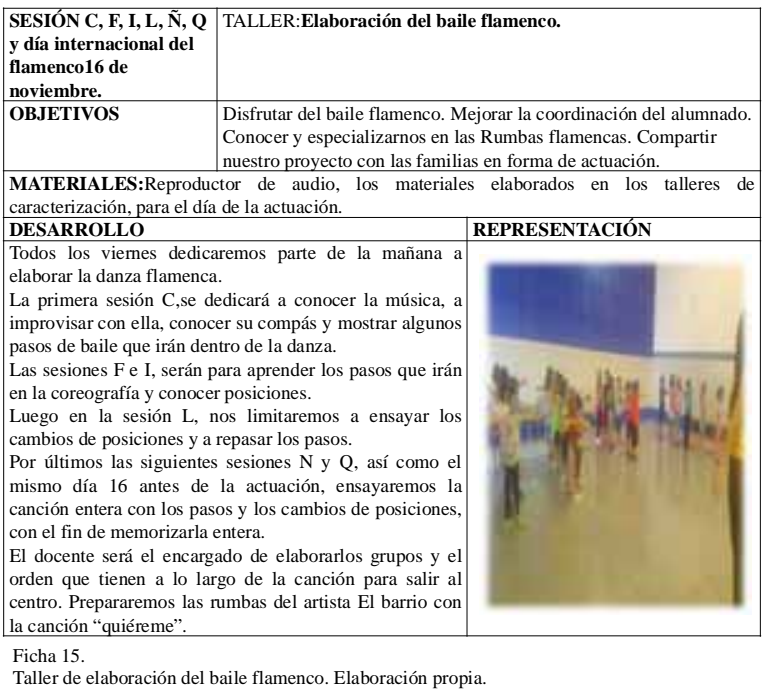

Taller de elaboración del baile flamenco. Elaboración propia.

\section{Decisiones de acción para la próxima puesta en práctica}

Para próximas intervenciones, consideramos que la propuesta se podría mejorar teniendo en cuenta:

-En la puesta en marcha de las actividades,utilizar imágenes que representen a cada una de ellas, como elemento motivador y recordatorio de lo aprendido, tal y como lo muestra Ibáñez-Granados (2015) en su propuesta didáctica para trabajar los componentes del movimiento.

- Integrar no solo a los padres en los talleres de reciclaje, sino en un mayor número de actividades, donde puedan compartir las experiencias con los niños.

- Trabajar de manera conjunta con los tutores de los grupos (Martín \& López, 2007)para mejorar el trabajo en equipo y cooperativo, ofreciéndoles la información necesaria sobre la importancia y los beneficios que aporta la danza o baile,para intentar que una de las razones de desaprovechamiento de éste recurso educativo no se produzca por desconocimiento(García, Pérez \& Calvo, 2011) y por el contrario se fomente su utilización en las aulas.

- Diseñar instrumentos de medida más objetivos para la recogida de datos quenos permitan medir los logros obtenidos con la aplicación del proyecto para elaborar mejoras y para el diseño de nuevos recursos o proyectos.

- Analizar la formación y el tiempo dedicado por los maestrosa la motricidad en el aula y comprobar si después de la aplicación del proyecto aumenta el interés por la utilización de estos recursos. 


\section{Conclusiones}

La utilización del baile flamenco como recurso motriz transversal, ha posibilitado la oportunidad de trabajar la motricidad durante más tiempo de lo que es habitual en el centro, lo que contribuirá a una mejora de la condición física y de las habilidades motrices básicas.

El alumnado ha sido capaz de adquirir conocimientos sobre los bailes flamencos, los diferentes accesorios utilizados por los bailaores y bailaoras, personajes relevantes del baile y del cante flamenco.

Las actividades les han permitido expresar mejor sus emociones, lo que contribuirá a un aumento de la autoestima y una mayor confianza en sí mismos.

La experiencia que ha vivido el alumnado ha sido una muestra de integración donde han reconocido, valorado y respetado otras culturas, favoreciendo las habilidades y relaciones sociales con sus compañeros a través del movimiento.

La participación de la familia ha supuesto un aumento en el interés y la motivación del alumnado hacia las actividades en las que estas han colaborado.

La utilización del baile flamenco como recurso educativo, puede tratarse dentro de las tres áreas del currículo de educación infantil (conocimiento de sí mismo y autonomía personal, en el conocimiento del entorno y en el lenguaje en su relación con la comunicación y representación), lo que pone de manifiestouna forma transversal de acercar al alumnado a las manifestaciones culturales y artísticas de Andalucía, fomentando el respeto e interés hacia las diferentes culturas y la propia,desarrollando competencias personales y sociales comoy mejorando la motricidad y el tiempo de práctica motriz del alumnado en el aula.

\section{Referencias}

Abilleira, M., Fernández-Villarino, M.A., Ramallo, S., \& Prieto, J.(2017). Influencia de la danza en el Autoconcepto del alumnado de Educación Primaria. Análisis comparativo con otras actividades físicas. Sportis, 3(3), 554-568.

BOJA (2014). Orden del 7 de Mayo de 2014, por las que se establecen medidas para la inclusión del flamenco en el sistema educativo andaluz. BOJA núm. 101, de 28 de Mayo de 2014, 17-27.

Cañal, F. \& Cañal, C. (2001). Música, danza y expresión corporal en educación infantil y primaria. Tomo 1. Junta de Andalucía-Consejería de Educación y Ciencia.

De las Heras \& Calderón-Garrido, (2018). El zapateado flamenco en las aulas: una revisión a la legislación. ArtesEduca, 19, 53-61.Recuperado de: http://www.erevistes.uji.es/index.php/artseduca/article/view/2794/2332

De Rueda, B., \& López, C.E. (2013). Música y programa de danza creativa como herramienta de expresión de emociones, Retos, Nuevas tendencias en Educación Física, Deporte y Recreación, 24, 141-148.

Decreto 428/2008, de 29 de julio, por el que se establece la ordenación y las enseñanzas correspondientes a la Educación Infantil en Andalucía. Boletín Oficial de la Junta de Andalucía, $n^{\circ}$ 164,2008, 19 agosto.

Duran, C. \& Costes, A. (2018). Efecto de los juegos motores sobre la toma de conciencia emocional/Effect of Motor Games on Emotional Awareness. Revista Internacional de Medicina y Ciencias de la Actividad Física y el Deporte, 18(70), 227-245

Esteve, A. I. \& López, V. M. (2014). La expresión corporal y la danza en educación infantil. La Peonza. Revista de Educación Física para La Paz, 9, 3-26.

Fuentes, A.L. (2006). El valor pedagógico de la danza. Valencia: Universidad de Valencia, Servicio de publicaciones.

García, D. \& Lizaso, B. (2012). Flamenco y nuevas tecnologías: el aula de música como contexto de integración del colectivo gitano. Publicaciones, 42, 1-20.

García, I., Pérez, R. \& Calvo, Á. (2011). Iniciación a la danza como agente educativo de la expresión corporal en la educación física actual. Aspectos metodológicos. Retos. Nuevas tendencias en Educación Física, Deportey Recreación, 20, 3336.

Gil-Espinosa, F.J., Romance, A.R. \& Nielsen, A. (2018). Juego y actividad física como indicadores de calidaden Educación Infantil. Retos, Nuevas tendencias en Educación Física, Deportey Recreación, 34, 252-257.

Ibáñez-Granados, D. (2015).Propuesta didáctica para trabajar los componentes del movimiento jugando a través de los cuentos, canciones, instrumentos musicales y rollitos de imágenes. Libro de actas de las II Jornadas «Educar jugando». Granada: Centro de Magisterio la Inmaculada.
Jefferson-Buchanan, R.(2012). Dance:Teaching and Learning Possibilities within the Early Years and Primary School Context. En G. Griggs (Ed.), An Introduction to Primary Physical Education (pp. 135-150). London: Routledge. Ley 17/2007, de 10 de diciembre de Educación deAndalucía.

Liu, J. (2017). Optimization of the combination of music education and preschool dance teaching based multimedia. Boletín técnico, 55(19), 117-122.

Lopez, M. A., Carlson, J. A., Cain, K. L., Bonilla, E. A., Chuang, E., Elder, J. P., \&Sallis, J. F. (2015). Dance Class StructureAffects Youth Physical Activity and Sedentary Behavior:AStudy of Seven Dance Types. Research Quarterly for Exercise and Sport, 86(3), 225-232.

Martín, M.I. \& López, V. (2007). Teatro de sombras en educación infantil: un proyecto para el festival de Navidad. Retos. Nuevas tendencias en Educación Física, Deporte y Recreación, 12, 45-50.

Moreno-Murcia, J.A.; Huéscar, E.; Polo, R.; López, E.; Carbonell, B. \& Meseguer, S. (2016). Efecto de los cuentos en la competencia acuática real y percibida en infantes/Tales Effect in Real and PerceivedAquatic Competence in Preschoolers. Revista Internacional de Medicina y Ciencias de la Actividad Física y el Deporte, 16(61),127-138.

Orden del 5 deAgosto de 2008, por la que se desarrolla el Currículo correspondiente a la Educación Infantil en Andalucía. Boletín Oficial de la Junta de Andalucía, $n^{\circ} 169,2008,26$ agosto.

Organización Mundial de Salud(2010). Recomendaciones Mundiales sobreActividad Física para la Salud. Recuperado de http:// apps.who.int/iris/bitstream/ 10665/44441/1/9789243599977_spa.pdf

Padial, R. \&Sáenz-López, P. (2014). Los Cuentos populares/tradicionales en educación infantil: una propuesta a través del juego. E-motion: Revista de Educación, Motricidad e Investigación 3, 32-47.

Pardo,A. \& Pacheco-Álvarez, M. (2015). Flamencoterapia: intervención alternativa para el alumnado con necesidades educativas especiales a través del baile flamenco. Revista de Investigación sobre el Flamenco La Madrugá,11, 115138. Recuperado de: http://revistas.um.es/flamenco

Renobell, S. (2009). Todo lo que hay que saber para bailar en la escuela. Barcelona: Inde.

Rial, T., \& Villanueva, C. (2016). El Flashmob como propuesta de innovación educativa en expresión corporal y danza. Retos. Nuevas tendencias en Educación Física, Deporte y Recreación, 29, 126-128.

Ríos, Y., Navarro, R., Arufe, V., \& Pérez, J.A. (2018). Evaluación de un programa de actividad física mediante juegos populares en escolares deEducación Primaria. Retos. Nuevas tendencias en Educación Física, Deporte y Recreación, 34, 108-113.

Rizo, G. (1996). La enseñanza de los bailes y las danzas tradicionales en la escuela: un enfoque interdisciplinar. Eufonía. Didáctica de la música, 3, 73-83.

Solís, A., Prieto, J.A., Nistal, P. \& Vázquez, Mª.M.(2017). Percepción y aplicación delapsicomotricidad por parte del profesorado de la etapa Infantil. Sportis, 3(1), 141-160.

Teixeira, H.J., Abelairas-Gomez, C., Arufe-Giráldez, V., Pazos, J.M., \& BarcalaFurelos, R. (2015). Influence of a physical education plan on psychomotor development profiles of preschool children. Journal Human Sport Exercise, 10 (1), 126-140.

Thom, L. (2010). From simple line to expressive movement: The use of creative movement toenhance socio-emotional development in the preschool curriculum. American Journal of Dance Therapy, 32(2), 100-112.

UNESCO (2010).El flamenco: Inscrito en 2010 (Decisión 5.COM 6.39) sobre la Lista Representativa del Patrimonio Cultural Inmaterial de la Humanidad. Recuperado de: https://ich.unesco.org/es/RL/el-flamenco-00363

UNESCO (2015). Educación Física de Calidad. Guía para los responsables políticos. Recuperado dehttp://unesdoc.unesco.org/images/0023/002313/231340s.pdf

Valdemoros-San Emeterio, Mª A., Sanz-Arazuri, E., Ponce-de-León-Elizondo,A. \& Alonso-Ruiz, R.A. (2018). Cualificación e implicación del profesorado de infantil frente a la educación motriz. Sportis , 4(1), 126-143.

Vicente, G., Ureña, N., Gómez, M. \& Carrillo, J. (2010). La danza en el ámbito de educativo. Retos. Nuevas tendencias en Educación Física, Deporte y Recreación, 17, 42-45.

Viciana, V., Cano, L., Chacón, R., Padial, R., \& Martínez,A. (2017). Importancia de la motricidad para el desarrollo integral del niño en la etapa de educación infantil. EmásF, Revista Digital de Educación Física, 47, Recuperado de: http://emasf.webcindario.com.

Zamorano, M.; Gil-Madrona, P.; Prieto-Ayuso, A. y Zamorano, D. (2018). Emociones generadas por distintos tipos de juegos en clase de educación física / Generated Emotions by Various Types of Games in Physical Education. Revista Internacional de Medicina y Ciencias de la Actividad Física y el Deporte, 18 (69), 1-26. 